\title{
Control of Surface Modes in Low Loss Hollow-Core Photonic Bandgap Fibers
}

\author{
R. Amezcua-Correa, F. Gérôme, S. Leon-Saval ${ }^{1}$, N. G. R. Broderick ${ }^{2}$, T. A. Birks and J.C. Knight \\ Centre for Photonics and Photonic Materials, Department of Physic ,University of Bath, Bath, BA2 7AY, United Kingdom \\ ${ }^{1}$ Now at the Optical Fibre Technology Centre, University of Sydney, NSW 2006, Australia \\ ${ }^{2}$ Optoelectronics Research Cetre, University of Southampton, Southampton SO17 1BJ, UK \\ r.a.correa@bath.ac.uk
}

\begin{abstract}
We report low-loss hollow-core photonic bandgap fibers free from surface modes. They have low attenuation over the full spectral width of the bandgap, and approximately halved dispersion and dispersion slope compared to previous fibers.

(C) 2008 Optical Society of America

OCIS codes: (060.2280) Fiber design and fabrication; (230.4000) Microstructure fabrication.
\end{abstract}

\section{Introduction}

Unlike conventional fibers that guide light by total internal reflection, in a hollow-core photonic bandgap fiber (HCPBGF) light is confined and guided by a photonic bandgap that prohibits the propagation of light in the cladding region under certain conditions [1]. Much effort in designing HC-PBGFs has been focused towards reducing the fiber attenuation, and impressive progress has been reported [2]. However, the performance of previously reported low loss HC-PBGFs has been limited by surface modes localized at the core cladding interface which couple to the core-guided mode, decreasing the useful bandwidth of the fibers and increasing the dispersion and dispersion slope.

\section{Fiber fabrication and optical properties}

Our fiber design is based on numerical computations which show that if the core walls have just half the thickness of the struts in the cladding then surface mode can be suppressed [3]. The fibers were fabricated from synthetic silica glass using the stack-and-draw technique. The stack was drawn in two drawing stages, with pressure used to inflate the structure. This process allows for easy and very rapid fabrication of fibers with core wall thickness close to the thickness required to eliminate surface modes, and having both broadband operation and state-of-the-art attenuation. The simplicity of the process makes it possible to draw a hollow-core fiber from scratch, within a single day. A typical SEM image of one of our canes before the final draw to fiber is shown in Fig. 1 (left).

Our thin core wall fiber, shown in Fig. 1(center), has a photonic crystal cladding with a pitch $\Lambda=6.7 \mu \mathrm{m}$, and air filling fraction of $96 \%$. For comparison, Fig. 1(right) shows a fiber similar to those commercially available (with state-of-the-art optical properties) for the same wavelength range - we will refer to it as "old fiber".
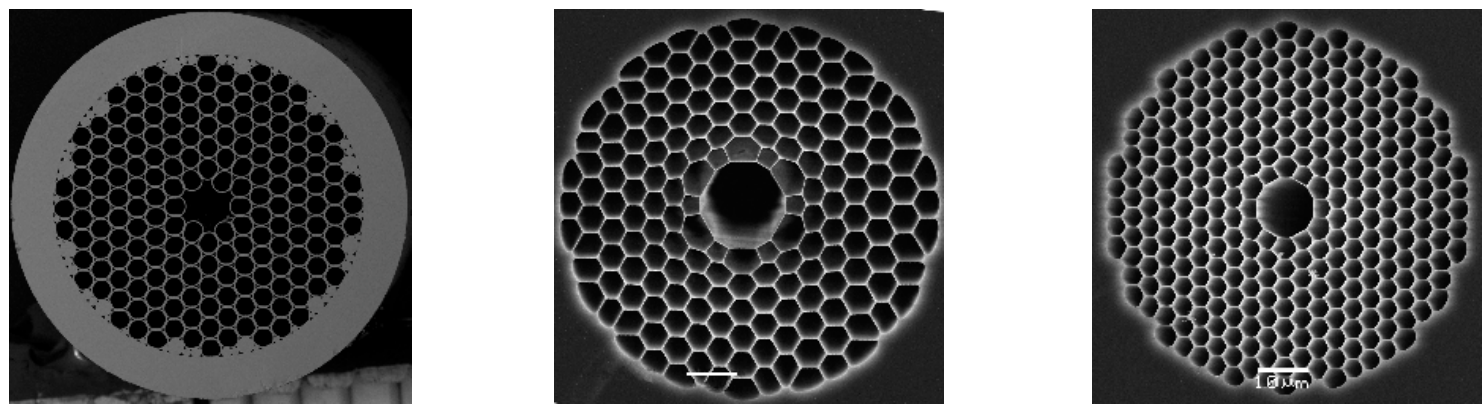

Fig. 1. Scaning Electron Micrographs (SEM). (left) A cane used to draw our 7-cell HC-PBGF, the outer diameter is $3.3 \mathrm{~mm}$. (center) Our fiber incorporating a thin core wall, and (right) standard HC-PBGF similar to that available commercially (old fiber). Scale bars correspond to $10 \mu \mathrm{m}$.

The optical attenuation of the thin core wall HC-PBGF was measured using the cut-back technique. The attenuation spectrum is shown in Fig. 2(black). The loss remains low over a wide bandwidth and there are no sharp peaks indicating the presence of surface mode anticrossings. The sharp increases in loss below $1450 \mathrm{~nm}$ and at around $1750 \mathrm{~nm}$ are due to the edges of the cladding bandgap. Low loss is achieved over a broad spectral range from $1450 \mathrm{~nm}$ to $1750 \mathrm{~nm}$, covering the full width of the photonic bandgap. The minimum attenuation is $15 \mathrm{~dB} / \mathrm{km}$ and remains below $50 \mathrm{~dB} / \mathrm{km}$ over approximately $300 \mathrm{~nm}$. This is the first time that light has been transmitted in a HCPBGF with low loss over such a wide spectral window. Mode profiles recorded at various wavelengths within the bandgap confirm that light propagates in a single mode which does not couple to surface modes, see Fig. 2(right). In contrast, the attenuation spectrum of the old fiber (Fig. 2 gray), centered at almost the same wavelength as our thin 
core wall fiber, presents distinct attenuation peaks. These high loss regions are due to surface mode anticrossings at $1450 \mathrm{~nm}$ and $1480 \mathrm{~nm}$. By eliminating the surface modes the effective bandwidth has been increased by approximately $70 \mathrm{~nm}$.

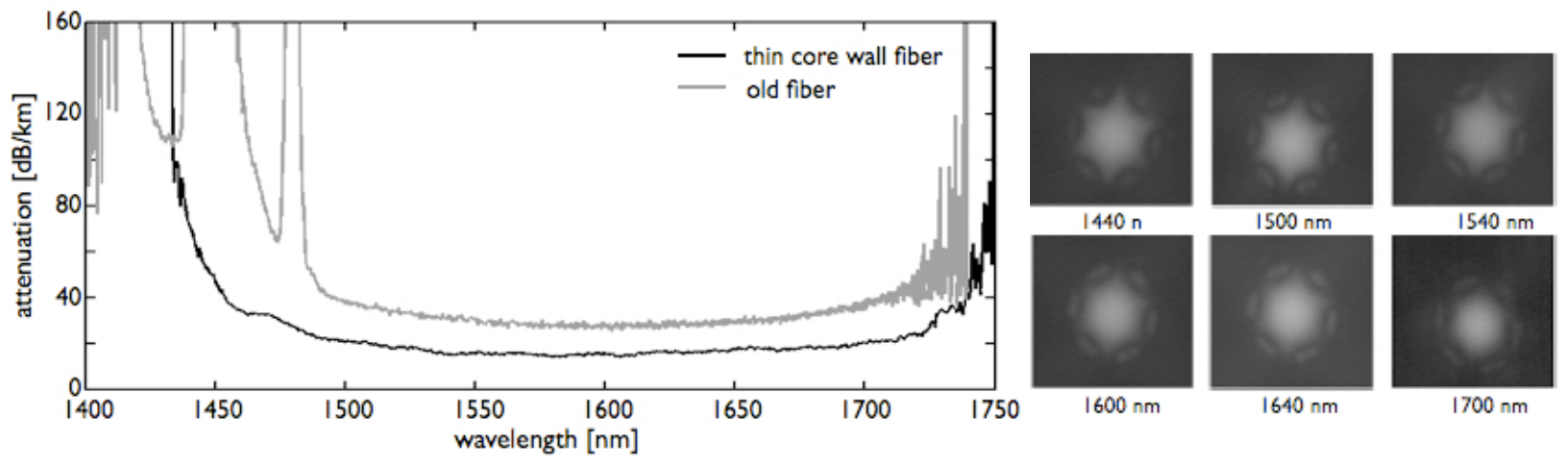

Fig. 2. Attenuation spectrum of (black) thin core wall fiber, and (gray) old fiber. (right) Near field images of the "fundamental" air-guided mode recorded after $50 \mathrm{~m}$ of the thin core wall fiber at various wavelengths within the bandgap.

The measured group velocity dispersion (GVD) for our fiber is given in Fig. 3(left), together with the transmission spectrum of $50 \mathrm{~m}$ of fiber. Neither the transmission spectrum nor the GVD show signs of surface mode anticrossings, resulting in smooth curves right across the bandgap. The GVD is $20 \mathrm{ps} / \mathrm{nm} / \mathrm{km}$ at the central wavelength. The GVD-slope is $0.3 \mathrm{ps} / \mathrm{nm}^{2} / \mathrm{km}$ over a very broad $200 \mathrm{~nm}$ spectral range from $1490 \mathrm{~nm}$ to $1690 \mathrm{~nm}$. This represents a factor of almost two reduction compared to previous state-of-the-art. Indeed, for the old fiber, dispersion at the center of the bandgap is $52 \mathrm{ps} / \mathrm{nm} / \mathrm{km}$ with a steeper GVD-slope of $54 \mathrm{ps} / \mathrm{nm}^{2} / \mathrm{km}$ over less than 130 nm, see Fig.3(right). It is worth noting that the third order dispersion of previously available HC-PBGFs has been a profound limitation on their performance for at least one important application: soliton compression of high power ultrashort pulses [4].
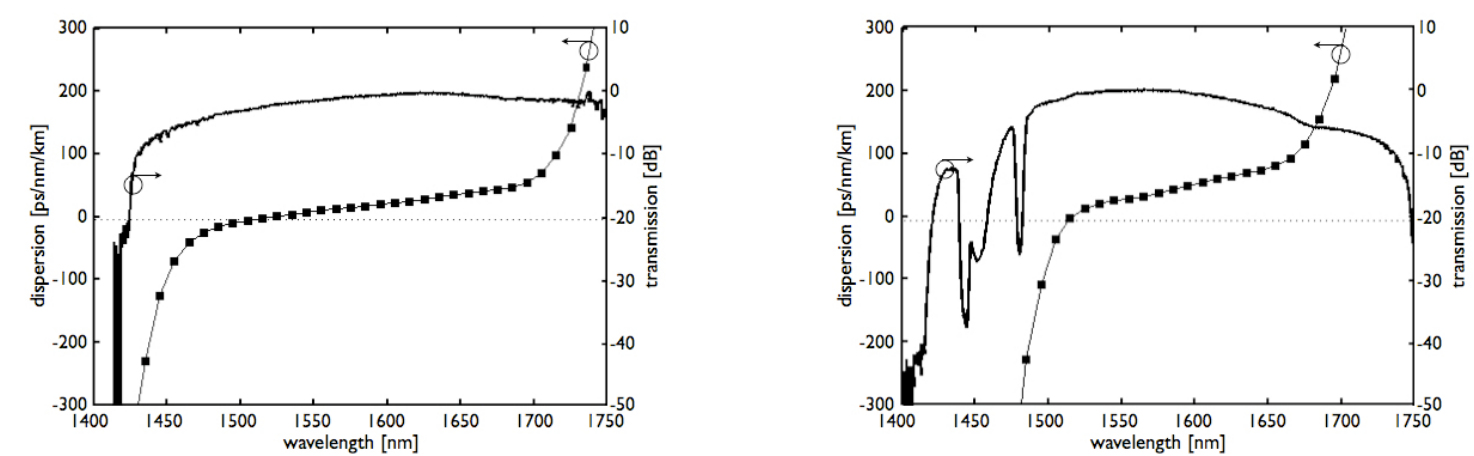

Fig. 3. Group velocity dispersion measured on $25 \mathrm{~cm}$ of fiber and normalized transmission through $50 \mathrm{~m}$ of fiber. (left) thin core wall fiber, and (right) old fiber.

\section{Conclusion}

We have demonstrated 7-cell HC-PBGFs with a novel core geometry incorporating a thin core/cladding interface. These fibers are able to guide light over a broader spectral window and with comparable or lower attenuation than previous state-of-the-art 7 cell HC-PBGFs. Fibers with minimum attenuation of $9.5 \mathrm{~dB} / \mathrm{km}$ at $1630 \mathrm{~nm}$ have also been fabricated. This is a consequence of the elimination of surface mode resonances within the bandgap. Our fibers also have the lowest dispersion slope yet reported for a HC-PBGF - a factor of almost two reduction compared to the prior state-of-the-art. Simplified fabrication of the new fibers enables the production of low-loss HC-PBGFs from scratch, within a single day. Extension of these designs to larger and smaller core sizes promises a range of hollowcore fibers with markedly improved performance compared to those previously reported.

This work was supported by the EU Framework 6 project "NextGenPCF", and the UK DTI and EPSRC.

[1] R.F. Cregan et al., "Single-mode photonic band gap guidance of light in air," Science 285, 1537-1539 (1999).

[2] J. A. West et al., "Surface modes in air-core photonic bandgap fibers," Opt. Express 12, 1485-1496, (2004).

[3] R. Amezcua-Correa et al., "Optimizing the usable bandwidth and loss in a hollow-core photonic bandgap fibre free of surface modes," Opt. Express. 14, 7974-7985 (2006).

[4] F. Gérôme et al., "Delivery of sub-100fs pulses through $8 \mathrm{~m}$ of hollow-core photonic crystal fiber using soliton compression," Opt. Express 15, 7126-7131 (2007). 